is, we think, that the leptomeningial arterial anastomosis plays the major role and there is no need of developing any "Chiri-chiri vessels".

From above mentioned findings, the "Chiri-chiri vessels" develop only when there is no effective collaterals to the area fo the mid. cereb. art. from the ant. and post. cereb. art. in which occlusion and stenosis affect the total course of the mid. cereb. and it's branches, and when these pathological process extend as for as the distal portion of the post. cereb. which angiographically looked patent but there exist stenosis or occlusion, as we had reported on an autopsied case in this congress, ' 66 .

And these "Chiri-chiri vessels" develop as collaterals to feed the area of the mid. cerebral art. Their original vessels would be the perforating arteries in the cerebral basal region which now dilated extremely to appear as "Chiri-chiri vessels".

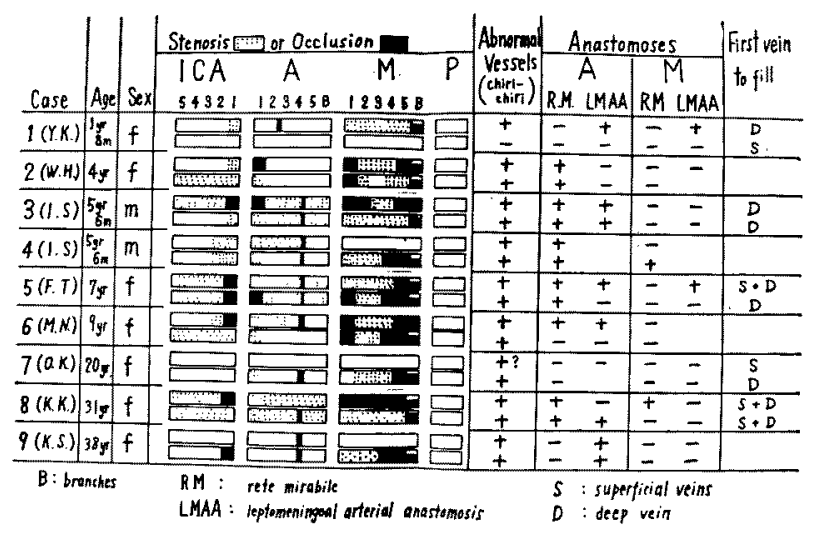

\title{
69. Some Trials of Surgical Treatment of the Disease showing Abnormal Vascular Network at the Base of the Brain
}

\author{
Jiro SUZUKI, Akira TAKaKu, Shigeharu SuzUKI, \\ Hiroshi HiguchI and Yoshiaki SaKuraI \\ Division of Neurosurgery, Institute of Brain Disease, Tohoku University \\ School of Medicine
}

We have postulated since 1963 that this disease is an acquired one which shows an abnormal vascular net work at the base of brain as a collateral pathways following stenosis or occlusion at the carotid fork.

In the 25th annual meeting of the Japan Neurosurgical Society, we also re- 
ported that this disease showed the noticeable dynamic changes on the follow-up study of carotid angiogram at least in young patients.

In this meeting, some trials of surgical treatment with the use of steroids and vasodilators for this disease were reported.

As the surgical treatment, perivascular sympathectomy of the carotid artery in the neck and our carotid dilatation method were performed at 6 carotid arteries in 3 cases of young patient and one adult case.

The operation was performed radically in five carotid arteries, so that their abnormal features in carotid angiogram changed for the better.

When no radical operation and no treatment were performed, their abnormal angiographic features showed a change for the worse or no improvement.

It was thought that this procedure would be effective for this disease.

\title{
70. Bilateral Carotid Artery Occlusion with Germinal Center in the Thymus: A Case Report
}

\author{
Ryuichi Kodama and Seiichi Mita \\ National Saitama Hospital \\ Kenichi Tamaoki and Tohoru AramaKi \\ Department of Pathology, Keio University \\ Yasuyoshi KIRYU and Keizo TAKAGI \\ Department of Medicine, Keio University \\ Hiroshi Yoshimatsu \\ Department of Surgery, Keio University
}

A case of bilateral carotid artery occlusion with abnormal vascular pattern, which is named to "Occlusion of the Willis circle" by Dr. Kudo, is reported. The 42 year old Japanese female admitted to National Saitama Hospital for trancient ischemic attack. The clinical cource and laboratory findings does not showed for the evidence of collagen diseases.

The pathogenesis of this disease is unknown, and we did peumomediastinography. The roentgenographic finding showed thymus enlargement. Thymus jiopsy is done. The thymus had had large and typical germinal center, just as patient of myasthnia gravis. This suggestive pathological finding may be related to the auto-immunologic process. 